\title{
Gesünderes Arbeiten im Wechselschichtdienst der Polizei in Rheinland-Pfalz - Anpassung der Schichtmodelle
}

\author{
Christine Watrinet ${ }^{1}$ S Silke Mündlein ${ }^{1} \cdot$ Jürgen Süs ${ }^{2} \cdot J^{\text {Jens }}$ Völlinger ${ }^{3}$ \\ Online publiziert: 19. November 2019 \\ (c) Der/die Autor(en) 2019
}

Schlüsselwörter Dienstplanung $\cdot$ Schichtarbeit $\cdot$ Wechselschichtdienst $\cdot$ Blockmodell $\cdot$ Bedarfsorientierung

\section{1 Überblick}

Die Polizei in Rheinland-Pfalz hat 2015 das Projekt „Gesünderes Arbeiten im Wechselschichtdienst" gestartet. Das Projekt sollte vor allem Werkzeuge zur Bewältigung der demografischen Veränderungen in der Belegschaft bereitstellen. Unter Berücksichtigung der dienstlichen Anforderungen des Polizeidienstes sowie der arbeitswissenschaftlichen Gestaltungsempfehlungen sollten die vollkontinuierlichen Arbeitszeitmodelle im Wechselschichtdienst lösungsorientiert neugestaltet werden. Darüber hinaus sollten die arbeitszeitrechtlichen Vorgaben zukunftsorientiert umgesetzt werden. Aus diesem Grund wurde in Rheinland-Pfalz 2015 eine Arbeitsgruppe „Gesünderes Arbeiten in der Polizei“ (AG GAP) gebildet, die den Auftrag hat, das Arbeiten im Polizeidienst gesünder zu gestalten und als erstes Handlungsfeld die Arbeitszeiten bzw. die Wechselschichtmodelle betrachtete.

Grundlage für diese herausfordernde Aufgabenstellung bildete ein partizipativer Prozess, der in dienststellenspezifischen Wechselschichtmodellen resultierte, die definierten Vorgaben entsprachen.

In 2017 wurden in mehreren Dienststellen verschiedene Modelle als Pilote getestet und evaluiert.

Der nachstehende Praxisbericht beschreibt das Projekt an sich, das Vorgehen und die zentralen Ergebnisse des Pilotjahres. Der Beitrag schließt mit den Empfehlungen und

\footnotetext{
Dr. Christine Watrinet

cw@ars-serendi.de

1 ars serendi GmbH, Herrenberger Straße 14, 71032 Böblingen, Deutschland

2 Polizeipräsidium Koblenz, Moselring 10-12, 56068 Koblenz, Deutschland

3 Polizeiinspektion Germersheim, Friedrich-Ebert-Straße 5, 76726 Germersheim, Deutschland
}

Regelungen, die ab 2019 von allen Dienststellen in Rheinland-Pfalz auf Basis einer Verwaltungsvorschrift umgesetzt werden.

Veranschaulicht werden die Ergebnisse in diesem Beitrag am Beispiel einer der Pilotdienststellen. Die Ergebnisse aller Dienststellen werden von Süs et al. (2018) in einem internen Arbeitspapier der Polizei Rheinland-Pfalz beschrieben.

\section{Ausgangslage, Zielsetzung und Rahmenbedingungen}

\subsection{Das Projekt „Gesünderes Arbeiten im Wechselschichtdienst"}

Die rheinland-pfälzische Polizei bewältigt derzeit große demografische Veränderungen. Die Beschäftigtenstruktur verändert sich massiv. Aufgrund von ca. 4440 Neueinstellungen in den Jahren 2009 bis 2018 ist die Polizei jünger und weiblicher geworden: Von den landesweit ca. 3600 Polizeibeamtinnen und -beamten (berechnet nach Vollzeitäquivalenten) in Wechselschicht waren $201523 \%$ weiblich und der Anteil derjenigen, die jünger als 40 Jahre sind, war bereits größer als der Anteil der über 40-jährigen. Gleichzeitig war die Anzahl der Polizeibeamtinnen und -beamten, die eingeschränkt dienstfähig waren, auf ca. 1000 angestiegen. Die Zahl der Fernpendler und -pendlerinnen hat mit der regionalen Einstellung durch die jeweiligen Polizeipräsidien bereits deutlich abgenommen. Diese Entwicklungen werden sich vermutlich weiter fortsetzen.

Diese Veränderungen führen zu neuen Bedürfnisstrukturen auf Seiten der Beschäftigten, die sich sowohl auf Aspekte der Arbeitgeberattraktivität als auch der Arbeitsfähigkeit auswirken. Als besonders relevante Einflussfaktoren wurden für die Polizei in Rheinland-Pfalz im Rahmen von Workshops mit den Beschäftigten folgende Punkte benannt: 
- die Möglichkeiten, die Arbeitszeit flexibel zu gestalten

- die Vereinbarkeit von Beruf und Privatleben

- die Arbeitsorganisation

- das Führungsverhalten

- die Formen der Zusammenarbeit

- das Arbeitsklima sowie die Organisationskultur

In puncto Arbeitszeitgestaltung ergab sich neben dem Wunsch nach flexiblen Arbeitszeiten weiterer Handlungsbedarf, da die praktizierten Modelle im Land zu einem groBen Teil nicht die gesetzlich erforderlichen Ruhezeiten von wenigstens $11 \mathrm{~h}$ vorsahen:

- 39 von 102 Dienststellen arbeiteten im sogenannten Doppelschlag. Der Doppelschlag sieht am ersten Tag des Schichtblockes eine Spätschicht, am folgenden Tag eine verkürzte Frühschicht und am Abend desselben Tages eine Nachtschicht vor. So werden innerhalb von ca. $42 \mathrm{~h}$ mindestens $24 \mathrm{~h}$ Dienst erbracht.

- Die Schichtlängen waren in einigen Dienststellen sowohl beim Doppelschlag als auch in Blockmodellen deutlich länger als mit acht Stunden vorgeplant.

- In einigen Dienststellen wurde durchgängig in 12-Stunden-Schichten gearbeitet.

Viele Beamte und Beamtinnen, auch in anderen Bundesländern, präferieren den Doppelschlag, da durch die Massierung der Arbeitszeit lange Freizeitblöcke entstehen. Die körperlichen Belastungen durch die langen Arbeits- und kurzen Ruhezeiten von weniger als elf Stunden sowie durch die Rückwärtsrotation werden von einem Großteil der Betroffenen bewusst zugunsten der längeren Freizeiten in Kauf genommen. Auch bei den $12 \mathrm{~h}$ Modellen wird Arbeitszeit massiert, um Freizeit zu optimieren.

Damit die Belastungen aus vollkontinuierlicher Schichtarbeit nicht zu übermäßigen individuellen Beanspruchungen führen, ist es Arbeitgeberpflicht, Schichtmodelle dem Schutzgedanken der Verhältnisprävention folgend arbeitswissenschaftlich gut zu gestalten. Bei einer vertraglichen wöchentlichen Arbeitszeit von 40 Wochenstunden ist dies eine besondere Herausforderung.

\subsection{Zielsetzung}

Zielsetzung des Projektes „Gesünderes Arbeiten im Wechselschichtdienst“" war es, ein Instrumentarium zu entwickeln, welches den Dienststellen in Rheinland-Pfalz ermöglicht, Wechselschichtmodelle zu gestalten, die zu ihren dienststellenspezifischen Bedarfen passen und gleichzeitig weniger belastend und sozial verträglicher sind.

Um diese übergeordnete Zielsetzung zu erreichen, wurden von der Arbeitsgruppe „Gesünderes Arbeiten in der Polizei“ (AG GAP) folgende Teilziele für das Projekt definiert:
1. Die arbeitswissenschaftlichen Empfehlungen werden berücksichtigt.

2. Die Vorgaben der Arbeitszeitgesetzgebung (RheinlandPfalz und EU-Richtlinie) werden eingehalten.

3. Es besteht weitestgehend Planungssicherheit im Wechselschichtdienst (WSD).

4. Die Leistungsfähigkeit und Effizienz des WSD werden idealerweise verbessert.

5. Es gibt keine Probleme bei Besetzung von Sondereinsätzen und Zusatzdiensten.

6. Der Einsatz zu sozial- und gesundheitsschädlichen Zeiten ist auf ein Mindestmaß reduziert.

7. Besondere Belastungen werden unmittelbar ausgeglichen.

8. Die Möglichkeit, Beruf und Privatleben zu vereinbaren, ist gegeben.

9. Eine subjektive Zufriedenheit mit den Modellen wird erreicht.

10. Die subjektiv eingeschätzte psychische und soziale Gesundheit bleibt erhalten oder wird besser.

Die Erreichung der Teilziele eins bis sieben wurde im Rahmen einer aufwändigen Analyse der tatsächlich gearbeiteten Dienstpläne überprüft, die übrigen drei Ziele wurden im Rahmen von Befragungen, d.h. durch die individuellen Bewertungen der Beamten und Beamtinnen, evaluiert.

\subsection{Arbeitswissenschaftlicher und rechtlicher Rahmen}

Die arbeitswissenschaftlichen und arbeitsmedizinischen Erkenntnisse, die in den neuen Wechselschichtmodellen berücksichtigt werden sollten, beruhen auf langjährigen Forschungsarbeiten und wissenschaftlicher Expertise. Ein guter Überblick findet sich bei Rothe et al. (2017) sowie auf den Seiten der Bundesanstalt für Arbeitsschutz und Arbeitsmedizin (BAuA) unter https://www.baua.de/DE/ Themen/Arbeitsgestaltung-im-Betrieb/Arbeitszeit/Nachtund-Schichtarbeit.html, Zugriff am 27.02.2018: „Nur ein gut organisierter Schichtplan minimiert die gesundheitlichen Risiken bei den (Beschäftigten) [...] und ermöglicht einen reibungslosen Ablauf [...]. Der Plan sollte ergonomisch sein und muss laut gesetzlicher Regelung nach arbeitswissenschaftlichen Erkenntnissen gestaltet sein.“

Ausgehend von den vorliegenden arbeitswissenschaftlichen Erkenntnissen formuliert die Bundesanstalt für $\mathrm{Ar}$ beitsschutz und Arbeitsmedizin folgende Handlungsempfehlungen (ebd.):

- Die Anzahl der aufeinanderfolgenden Nachtschichten sollte möglichst gering sein. 
- Nach einer Nachtschichtphase sollte eine möglichst lange Ruhephase folgen. Sie sollte auf keinen Fall weniger als $24 \mathrm{~h}$ betragen.

- Geblockte Wochenendfreizeiten sind besser als einzelne freie Tage am Wochenende.

- Schichtarbeiter sollten möglichst mehr freie Tage im Jahr haben als Tagarbeiter.

- Ungünstige Schichtfolgen sollten vermieden werden, das heißt immer vorwärts rotieren.

- Die Frühschicht sollte nicht zu früh beginnen.

- Die Nachtschicht sollte möglichst früh enden.

- Zugunsten individueller Vorlieben sollte auf starre Anfangszeiten verzichtet werden.

- Die Massierung von Arbeitstagen oder Arbeitszeiten auf einen Tag sollte begrenzt werden.

- Schichtpläne sollen vorhersagbar und überschaubar sein.

Ebenso wichtig ist es, die Länge der Schichten der Arbeitsbelastung anzupassen. Schichten mit einer Länge von neun und mehr Stunden sind arbeitswissenschaftlich kritisch zu sehen. Untersuchungen von Spencer et al. (2006) weisen darauf hin, dass das Risiko für Unfälle und Fehlhandlungen $\mathrm{ab}$ der neunten Stunde überdurchschnittlich steigt.

Ein weiteres wichtiges Ziel des Projektes war und ist die Umsetzung von geltendem Recht. Die Arbeitszeitmodelle für den Wechselschichtdienst müssen den Regelungen der aktuellen Arbeitszeitgesetzgebung entsprechen. Die EU-Arbeitszeitrichtlinie (2003) enthält Mindestvorschriften für Sicherheit und Gesundheitsschutz bei der Arbeitszeitgestaltung; die Arbeitszeitverordnung des Landes RheinlandPfalz fügt sich in diesen Schutzgedanken ein. Daher sind in den neuen Wechselschichtmodellen folgende Vorgaben einzuhalten:

- Tägliche zusammenhängende Ruhezeit von $11 \mathrm{~h}$ pro $24-$ Stunden-Zeitraum

- Wöchentliche zusammenhängende Ruhezeit von $36 \mathrm{~h}$ pro 7 Tage-Zeitraum (Die Arbeitszeitverordnung RheinlandPfalz ist bezüglich dieses Punktes enger gefasst als die EU-Arbeitszeitrichtlinie.)

- Begrenzung der Nachtarbeit auf $8 \mathrm{~h}$ pro 24-Stunden-Zeitraum

- Begrenzung der wöchentlichen Höchstarbeitszeit auf $48 \mathrm{~h}$ im viermonatigen Ausgleichszeitraum

\section{Vorgehen}

\subsection{Partizipation und Auswahl der Pilotdienststellen}

Projekte, die sich mit der Veränderung von Arbeitszeitmodellen beschäftigen, sind hoch emotionale Prozesse, in denen es gilt, eine größtmögliche Schnittmenge zwischen den Interessen der Beschäftigten, der Organisation und des rechtlichen sowie arbeitswissenschaftlichen Rahmens aktiv zu gestalten. Dies gelingt gut, wenn ein gemeinsamer Kriterienkatalog für die Bewertung alternativer Arbeitszeitmodelle entwickelt wird, sodass die Gestaltung der neuen Modelle für alle nachvollziehbar und transparent wird.

Um die Erwartungen der Beschäftigten für den Kriterienkatalog abzufragen, wurden Workshops und Informationsveranstaltungen durchgeführt.

Laut BAuA (können und) müssen die beschriebenen arbeitswissenschaftlichen „Empfehlungen nicht alle gleichzeitig erfüllt und auch nicht nach der obigen Reihenfolge umgesetzt werden. Vielmehr müssen die einzelnen Kriterien individuell bewertet werden und an die Anforderungen [...] (der jeweiligen Organisation bzw. Dienststelle) angepasst werden." (BAuA).

Daher priorisierte die Arbeitsgruppe die Empfehlungen für die Gestaltung der Schichtmodelle für die Pilotphase des Projektes „Gesünderes Arbeiten im Wechselschichtdienst“ unter Berücksichtigung der Bedarfe der Kollegen und Kolleginnen (Wichtigkeit in absteigender Reihenfolge):

1. Freizeit in möglichst langen Blöcken

2. nicht mehr als drei Nachtschichten hintereinander

3. maximal fünf bis sieben aufeinanderfolgende Arbeitstage

4. gesicherte Wochenendfreizeiten

5. schnelle Vorwärtsrotation (Wechsel von Früh- auf Spätund dann auf Nachtschicht)

6. Länge der Schichten der Arbeitsbelastung anpassen

Bei der Bildung der Arbeitsgruppe wurde darauf geachtet, dass alle Polizeipräsidien, Funktionseinheiten und Interessensgruppen gleichermaßen eingebunden wurden.

Für die einjährige Pilotphase in 2017 wurden in einem aufwändigen Informations- und Bewerbungsverfahren sieben Dienststellen als sogenannte Hauptpiloten ausgewählt und sechs weitere Dienststellen als sogenannte Nebenpiloten zugelassen.

Bei der Auswahl der Hauptpilotdienststellen wurde darauf Wert gelegt, dass alle Präsidien, unterschiedlich große Dienststellen und vor allem möglichst verschiedene Wechselschichtmodelle berücksichtigt wurden. Voraussetzung für die Teilnahme war ergänzend eine Zustimmung der Beamtinnen und Beamten der Dienststelle für die Pilotierung von wenigstens $65 \%$.

Im Folgenden liegt der Fokus auf den Hauptpiloten, die Erfahrungen der Nebenpilotdienststellen, die eine Selbstevaluation durchführten, bestätigen die Ergebnisse aus den Hauptpilotdienststellen weitestgehend. Die Hauptpilotdienststellen unterzogen sich einer Fremdevaluation.

Die Pilotdienststellen wurden bei der Gestaltung der Pilotmodelle durch die AG GAP beraten und entschieden dann eigenverantwortlich, welches Modell getestet werden 
sollte. So wurde nicht immer das objektiv beste Modell hinsichtlich Bedarf und Verfügungsstärke gewählt. Dies wurde bewusst zugelassen, da die Mitgestaltung und die Berücksichtigung individueller Bedürfnisse für die Zufriedenheit in Veränderungsprozessen die Arbeitszeit betreffend wichtige Einflussgrößen sind.

\subsection{Vom Doppelschlag zum Blockmodell}

Wie oben beschrieben, ist der sogenannte Doppelschlag ein bevorzugtes Modell. Daher lag ein besonderes Augenmerk auf Pilotdienststellen, die aus dem Doppelschlag kommend, ein arbeitswissenschaftlich empfehlenswertes Blockmodell im Pilotjahr getestet haben. Dieses trifft für die Pilotdienststelle zu, deren Pilotergebnisse in diesem Beitrag näher betrachtet werden (vgl. Tab. 1).

In der hier vorgestellten Pilotdienststelle, mit einer Schichtbelegschaft von ca. 50 bis 55 Beamten und Beamtinnen, die in der Pilotphase regelmäßig im Wechsel-

Tab. 1 Doppelschlag, 39 der 102 Polizeiinspektionen in RheinlandPfalz arbeiteten in diesem Modell, der Plan wiederholt sich nach fünf Wochen, Schichtwechselzeiten $\mathrm{f}=$ Frühschicht Dauer von 6 bis $13 \mathrm{Uhr}, \mathrm{s}=$ Spätschicht Dauer von 12 bis $21 \mathrm{Uhr}, \mathrm{n}=$ Nachtschicht Dauer von 21 bis 6 Uhr, Überlappzeit zwischen Früh- und Spätschicht von einer Stunde

\begin{tabular}{|c|c|c|c|c|c|c|c|}
\hline $\begin{array}{l}\text { Wochen im } \\
\text { Schichtplan, } \\
\text { die eine Schicht- } \\
\text { gruppe durchläuft }\end{array}$ & Mo & $\mathrm{Di}$ & $\mathrm{Mi}$ & Do & $\mathrm{Fr}$ & $\mathrm{Sa}$ & So \\
\hline 1 & $\mathrm{~s}$ & $\mathrm{f} / \mathrm{n}$ & - & - & - & $\mathrm{s}$ & $\mathrm{f} / \mathrm{n}$ \\
\hline 2 & - & - & - & $\mathrm{s}$ & $\mathrm{f} / \mathrm{n}$ & - & - \\
\hline 3 & - & $\mathrm{s}$ & $\mathrm{f} / \mathrm{n}$ & - & - & - & $\mathrm{s}$ \\
\hline 4 & $\mathrm{f} / \mathrm{n}$ & - & - & - & $\mathrm{s}$ & $\mathrm{f} / \mathrm{n}$ & - \\
\hline 5 & - & - & $\mathrm{s}$ & $\mathrm{f} / \mathrm{n}$ & - & - & - \\
\hline
\end{tabular}

Tab. 2 Blockmodell mit sechstägigen Schichtblöcken, der Plan wiederholt sich nach zehn Wochen, Schichtwechselzeiten $\mathrm{f}=$ Frühschicht Dauer von 6 bis 14 Uhr, $\mathrm{s}=$ Spätschicht Dauer von 14 bis $22 \mathrm{Uhr}, \mathrm{n}=$ Nachtschicht Dauer von 22 bis $6 \mathrm{Uhr}$

\begin{tabular}{llllllll}
\hline $\begin{array}{l}\text { Wochen im } \\
\text { Schicht- } \\
\text { plan, die eine } \\
\begin{array}{l}\text { Schichtgruppe } \\
\text { durchläuft }\end{array}\end{array}$ & Mo & Di & Mi & Do & Fr & Sa & So \\
\hline 1 & f & f & s & s & n & n & - \\
2 & - & - & - & f & f & s & s \\
3 & n & n & - & - & - & - & f \\
4 & f & s & s & n & n & - & - \\
5 & - & - & f & f & s & s & n \\
6 & n & - & - & - & - & f & f \\
7 & s & s & n & n & - & - & - \\
8 & - & f & f & s & s & n & n \\
9 & - & - & - & - & f & f & s \\
10 & s & n & n & - & - & - & - \\
\hline
\end{tabular}

schichtdienst arbeiteten, kam während der Pilotphase ein kurz vorwärts rotierendes Blockmodell mit sechstägigen Schichtblöcken (vgl. Tab. 2) zum Einsatz. Dieses Blockmodell entspricht den arbeitswissenschaftlichen Empfehlungen und dem rechtlichen Rahmen ohne Einschränkungen. Die Veränderungen bei der Anzahl an Beamten und Beamtinnen im Jahresverlauf resultieren aus $\mathrm{Zu}$ - und Wegversetzungen zu den üblichen Versetzungsterminen im Mai und Oktober eines Jahres.

Diese Dienststelle wurde für die Darstellungen in diesem Bericht ausgewählt, da hier ,,aus dem Doppelschlag kommend“ zum einen das gleichmäßige Blockmodell getestet wurde und zum anderen war diese Dienststelle die einzige, die eine Belastungsanalyse durchgeführt hat.

Das bedeutet, über das Jahr wurde über die Tage stundenweise genau der Bedarf an Beamten und Beamtinnen bzw. deren Tätigkeiten im Dienst analysiert, um den Dienstplan am wirklichen Bedarf orientiert zu gestalten. Ziel war festzustellen, ob gerade zu den sozial- und gesundheitsschädlichen Zeiten in der Nacht und an den Wochenenden die Personalstärke reduziert werden kann. Im Ergebnis zeigte sich, dass es möglich ist, an bestimmten Wochentagen die Nachtschichten für Einzelne vorzuziehen oder zu verkürzen, d.h. die Nachtschicht bereits zwischen 2 und $4 \mathrm{Uhr}$ zu beenden, sodass ein Schlaf zur Nachtzeit noch ermöglicht wird.

Die Abb. 1 zeigt das durchschnittliche Einsatzaufkommen in der Pilotdienststelle im Tagesverlauf unabhängig vom Wochentag so wie die Schichtzeiten von Früh-, Spätund Nachtschicht (Zeitraum 01.09.2016 bis 31.12.2017). Sich daraus ergebende Möglichkeiten für versetzte Dienste zur Förderung der Vereinbarkeit von Beruf und Familie und des Gesundheitsschutzes bzw. der entsprechende Zeitraum in dem dieses besonders gut möglich ist, wird durch den abgebildeten Rahmen angezeigt. Soweit es die Einsatzlage und Besetzungsstärke zulässt, arbeiten beispielsweise jeweils zwei von elf Beamten und Beamtinnen einer Schichtgruppe versetzt. Häufig wird dies durch einen vorgezogenen Nachtdienst realisiert, der von ca. 18 bis 2 Uhr dauert. So können die Kollegen und Kolleginnen der Spätschicht unterstützt und der Nachtschlaf für einzelne verbessert werden. Die versetzten Dienste werden flexibel, orientiert an dienstlichen Anforderungen und individuellen Bedürfnissen gestaltet.

Beim Wechsel vom Doppelschlag auf ein Blockmodell mit gleichmäßig sechstägigen Schichtblöcken und viertägigen Freiblöcken lauteten die zentralen Fragestellungen für die Evaluation der Pilotphase wie folgt:

- Werden die viertägigen Erholungszeiten nach den sechstägigen Schichtblöcken als ausreichend wahrgenommen?

- Wird eine Entlastung durch die kürzeren Spät- und Nachtdienste von $8 \mathrm{~h}$ empfunden? 
Abb. 1 Belastungsanalyse über den Tagesverlauf in der Pilotdienststelle

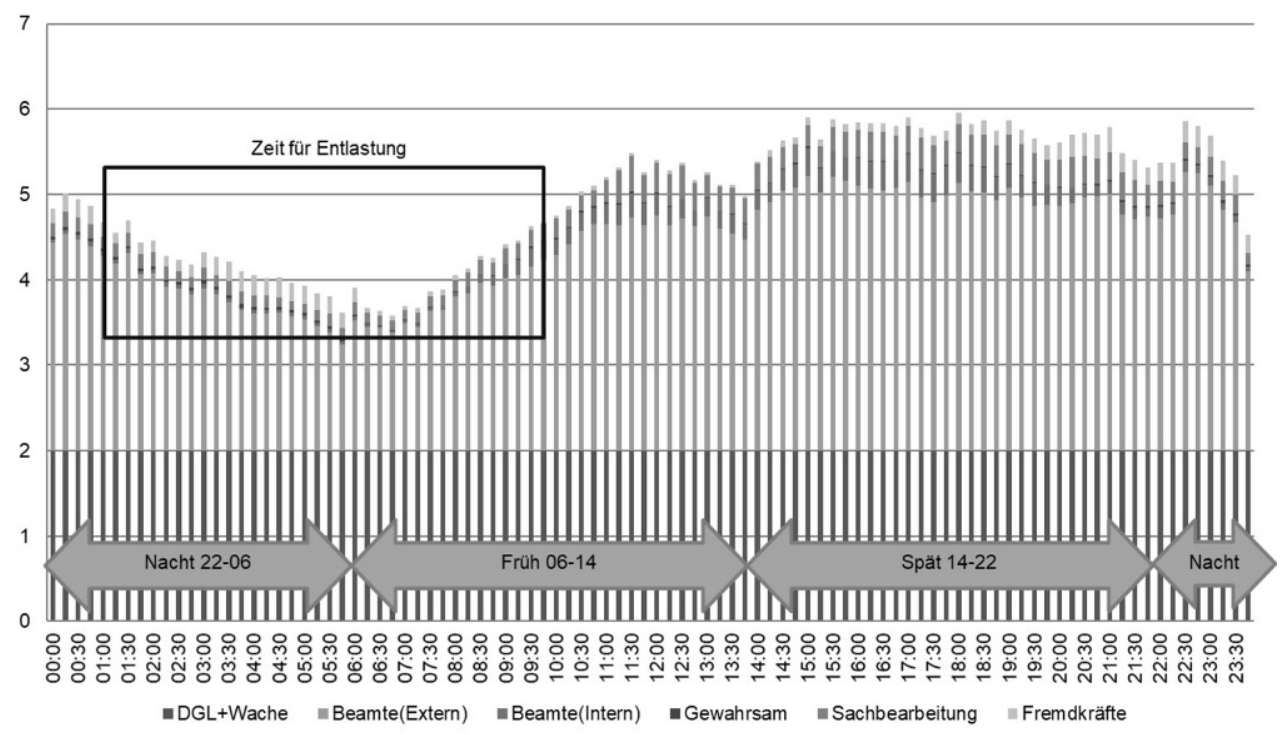

- Wird das Blockmodell im Hinblick auf Beanspruchung, Gesundheit, Vereinbarkeit von Beruf und Privatleben etc. gut bewertet?

\section{Bausteine der Evaluation}

\subsection{Inhalt und Methodik der Fragebögen}

Um Antworten auf die zentralen Fragestellungen des Projektes zu erhalten, setzte sich die Evaluation der Hauptpiloten aus mehreren Bausteinen zusammen. Kern der Evaluation waren drei schriftliche Befragungen. Die Befragungen wurden ergänzt durch ein Monitoring der Schichtpläne im Pilotverlauf.

Die drei Fragebögen wurden entwickelt auf Basis

- einer umfangreichen Analyse der Ausgangssituation, in deren Rahmen die Struktur der Dienststellen im Land beschrieben wurde,

- der beschriebenen Zielsetzungen, die von der Arbeitsgruppe definiert wurden

- und der spezifischen Zielsetzungen der an der Pilotphase teilnehmenden Dienststellen bzw. den aus den Modellwechseln resultierenden Wirkungen.

Der erste Fragebogen diente zur Bewertung des ,alten“ Schichtmodells vor Start in das Pilotjahr, zwei weitere Instrumente wurden für die Zwischenbefragung und die Abschlussbefragung eingesetzt. Die Zeitpunkte der Befragungen waren wie folgt:

- Auftaktbefragung: 06.12.2016-13.01.2017

- Zwischenbefragung: 06.06.-02.07.2017

- Abschlussbefragung: 28.11.-22.12.2017
Folgende Konstrukte wurden in den Fragebögen abgebildet:

- Schichtbiografie und soziodemografische Daten

- Bewertung des Piloten insgesamt

- Wohlbefinden, Erholungs- und Leistungsfähigkeit

- Umfang der Arbeitszeit

- Länge von Arbeits- und Freiblöcken, Anfangszeiten, Änderungen im Dienstplan und Umgang mit Anholungen zu Sonder-/Ergänzungs- und Zusatzdiensten

- arbeitsorganisatorische Regelungen auf der Dienststelle

- der Umgang miteinander und Führung; Kultur- und Klimaaspekte

- Schlafqualität und -quantität

- körperliche, psychische und zeitliche Beanspruchung

- Belastung in unterschiedlichen Schichten an den verschiedenen Wochentagen

- Unterstützungsangebote und Betriebliches Gesundheitsmanagement (BGM)

- Arbeitsfähigkeit mittels Einsatz des Work Ability Index in Auftakt- und Abschlussbefragung

Theoretische Grundlage für die ausgewählten Konstrukte sind die Ergebnisse vorausgehender Arbeiten (u.a. Knauth und Hornberger 1987; Rutenfranz et al. 1993; Bürger 2015; DGUV Report 1/2012). Das heißt, berücksichtigt wurden vor allem die mehrfach empirisch bestätigten arbeitswissenschaftlichen Erkenntnisse sowie die subjektiven Empfindungen der Betroffenen. Der subjektiven Wahrnehmung der Beamtinnen und Beamten im Wechselschichtdienst kommt eine wesentliche Rolle zu, wenn die (individuelle) Beanspruchung eines Schichtmodells zuverlässig abgebildet werden soll (Nachreiner 1978).

Die Items im Fragebogen wurden in der Regel mittels einer fünfteiligen Likert-Skala bewertet. Die Antwortmög- 
lichkeiten reichten von ,die Aussage trifft voll und ganz zu“ bis zu ,die Aussage trifft überhaupt nicht zu“. Zusätzlich gab es eine sechste Antwortalternative: „die Aussage kann nicht eingeschätzt werden“".

Ergänzt wurden die Ankreuzmöglichkeiten durch Freitextfelder, in denen die Beamtinnen und Beamten weitere Rückmeldungen eintragen konnten.

Um die Daten der verschiedenen Befragungen bei gleichzeitiger Wahrung der Anonymität zuverlässig miteinander vergleichen zu können, wurde ein bewährtes Verfahren zur Codierung genutzt. Die Beamtinnen und Beamten wurden gebeten, anhand einer Mustervorlage einen Code aus zwei Buchstaben und vier Ziffern zu bilden und diesen zu jedem Befragungszeitpunkt auf den Bögen zu notieren.

\subsection{Auswertung}

Die Auswertungen wurden mit SPSS 25 durchgeführt. Um die möglichen Veränderungen im Zeitverlauf zwischen den drei abhängigen Befragungen für die Gesamtstichprobe von 246 Teilnehmenden an der Auftaktbefragung, 253 in der Zwischenbefragung und 217 in der Abschlussbefragung beurteilen zu können, wurden einfaktorielle Varianzanalysen (mit Messwiederholung) und Post-hoc-Tests mit Bonferroni-Korrektur durchgeführt. Fehlende Daten wurden fallweise ausgeschlossen. Das Signifikanzniveau wurde gegen $p<0,05$ getestet.

Da die Stichproben in den einzelnen Dienststellen aufgrund ihrer Größe und der zum Teil schiefen Verteilungen nicht die Voraussetzungen für eine einfaktorielle Varianzanalyse oder den T-Test erfüllten, kam hier der WilcoxonVorzeichen-Rang-Test zum Einsatz. Dieser Test war sinnvoll, da in den meisten Dienststellen nur ein Teil der Probanden an allen drei Befragungen teilgenommen hat, andere nur an der ersten und zweiten oder an der ersten und dritten Befragung. So wurden diese Messzeitpunkte jeweils ins Verhältnis gesetzt. Auch in diesem Fall wurde gegen das Signifikanzniveau $p<0,05$ getestet.

Um weitere offensichtliche (Einfluss-)Faktoren auf das gesündere Arbeiten im Wechselschichtdienst beurteilen zu können, wurde eine Hauptkomponentenanalyse durchgeführt. Die sieben identifizierten Faktoren erklären knapp $62 \%$ der Gesamtvarianz. Für die Faktorenanalyse wurde eine reduzierte Itemanzahl genutzt, damit die Fallzahl in etwa dreimal so groß wie die Itemanzahl ist. Die Auswahl der Items erfolgte anhand der deskriptiven Analyse, den beobachteten Signifikanzen und sachlogischen Überlegungen.

Ergänzend zu den Befragungen wurden die Schichtpläne der Pilotdienststellen ausgewertet. Es erfolgte ein detaillierter Soll-Ist-Abgleich zu Umfang und Lage der tatsächlich erbrachten Schichten. So konnte beurteilt werden, ob auch die individuell mit Zusatzschichten ergänzten Pläne nach wie vor den beschriebenen Teilzielen entsprachen bzw. diese erfüllen konnten.

\section{Ergebnisse}

\subsection{Stichprobengröße und Darstellung der Ergebnisse}

Wie schon beschrieben nahmen an der Pilotphase an den Befragungen zwischen 217 und 253 Beamte und Beamtinnen aus sieben Pilotdienststellen teil. Die Qualität der Daten war in allen Pilotdienststellen sehr gut: Die Fragebögen wurden mehrheitlich vollständig ausgefüllt, es gab kaum fehlende Antworten. Eine Dienststelle nahm an der Abschlussbefragung nicht mehr teil, daher war die Gesamtstichprobe in der Abschlussbefragung deutlich kleiner. Diese Dienststelle brach den Piloten nach der Zwischenbefragung ab, da das für die Pilotphase gewählte Modell nicht zu den Bedarfen der Dienststelle passte. Wie schon beschrieben, wurde die Auswahl der Pilotmodelle weitestgehend den Dienststellen selbst überlassen, um das Gefühl ,übergestülpter" Dienstmodelle zu vermeiden.

Exemplarisch werden die Ergebnisse der Pilotphase im Folgenden anhand der einen bereits erwähnten Hauptpilotdienststelle dargestellt. In dieser hier näher betrachteten Dienststelle waren in der Pilotphase rund 50 Beamte und Beamtinnen beschäftigt. Es handelt sich um eine sogenannte mittelgroße Dienststelle, deren Beschäftigtenstruktur die Entwicklungen im Polizeidienst in Rheinland-Pfalz gut widerspiegelt. Die Teilnahmequote betrug in dieser Dienststelle nahezu an allen Befragungen wenigstens $90 \%$.

Die Tab. 3 zeigt den Rücklauf der Fragebögen aus dieser Pilotdienststelle. An allen drei Befragungen nahmen 26 Beamte und Beamtinnen teil, sechs nahmen nur an der Auftakt- und Zwischenbefragung und einer oder eine nur an Auftakt- und Abschlussbefragung. Diese 33 Datensät-

Tab. 3 Rücklauf in der hier betrachteten Pilotdienststelle und Beteiligung an den verschiedenen Befragungen auf Basis der angegebenen Codierung. Fragebögen mit fehlender Codierung wurden bei der Betrachtung der wiederholten Teilnahme an den verschiedenen Befragungen nicht berücksichtigt

Rücklauf ausgewählte Pilotdienststelle

Anzahl ausgefüllter Fragebögen

(in \% von den ausgeteilten Fragebögen)

\begin{tabular}{ll}
\hline Auftaktbefragung & $50(100 \%)$ \\
Zwischenbefragung & $54(98,1 \%)$ \\
Abschlussbefragung & $55(90,9 \%)$ \\
an allen drei Befragungen & $26(52 \%$ von 50 der Auftaktbefragung) \\
Auftakt und Zwischen & $6(12 \%$ von 50 der Auftaktbefragung) \\
Auftakt und Abschluss & $1(2 \%$ von 50 der Auftaktbefragung) \\
Zwischen und Abschluss & $\begin{array}{l}11(20,4 \text { von 54 der Zwischenbefra- } \\
\text { gung) }\end{array}$ \\
\hline
\end{tabular}


ze wurden für die Überprüfung der signifikanten Veränderungen im Pilotjahr genutzt. Der Anteil derjenigen, die nachweislich an der Auftakt- und einer weiteren Befragung teilnahmen ist so gering, da die Codierung häufig nicht eingetragen wurde.

Für die Darstellung der Ergebnisse der Dienststelle insgesamt wurden alle Daten der jeweiligen Befragungen berücksichtigt, da in der Praxis für die Bewertung von Wechselschichtmodellen jede Meinung wichtig ist.

Die Ergebnisse werden anhand von Mittelwertvergleichen und Häufigkeiten abgebildet. Zunächst werden die Ergebnisse bezüglich der oben beschriebenen, durch die Projektgruppe ausgewählten, relevanten arbeitswissenschaftlichen Kriterien abgebildet. Nachfolgend zu diesen Aspekten wird die Zufriedenheit mit den Modellen bezüglich der Vereinbarkeit von Beruf und Privatleben sowie der Einschätzung von Gesundheit und Arbeitsfähigkeit beschrieben.

Die Ergebnisse zeigen, dass das pilotierte Schichtmodell für diese Dienststelle optimal war. Die Dienststelle arbeitete in diesem Modell auch nach der Pilotphase in 2018 weiter.

\subsection{Bewertung anhand der arbeitswissenschaftlichen Empfehlungen}

Die beiden sich gegenseitig bedingenden Kriterien, möglichst lange Freiblöcke und maximal fünf bis sieben aufeinanderfolgende Arbeitstage zu planen, wurden zusammengefasst.

1. Freizeit in möglichst langen Blöcken, dennoch maximal fünf bis sieben aufeinanderfolgende Arbeitstage

2. nicht mehr als drei Nachtschichten hintereinander

3. gesicherte Wochenendfreizeiten

4. schnelle Vorwärtsrotation (Wechsel von Früh- auf Spätund dann auf Nachtschicht)

5. Länge der Schichten der Arbeitsbelastung anpassen

\subsubsection{Freizeit in möglichst langen Blöcken, dennoch maximal fünf bis sieben aufeinanderfolgende Arbeitstage}

Das Bedürfnis, in Schichtarbeit möglichst lange Freiblöcke zu generieren, korrespondiert mit dem Ziel, die Schichtlänge der Blöcke zu minimieren. Der Lösungsraum ist endlich: In den pilotierten Blockmodellen auf Basis von fünf Dienstgruppen gilt es, 21 Schichten mit in der Regel einer Länge von acht Stunden und entsprechend 14 freie Tage innerhalb von fünf Wochen belastungsorientiert zu verteilen.

Wird dabei eine schnelle Vorwärtsrotation der Schichten umgesetzt (maximal zwei bis drei Schichten einer Art hintereinander), enden die Schichtblöcke jeweils mit einer oder zwei Nachtschichten. Nach den Nachtschichten sind zwingend $24 \mathrm{~h}$ frei zu gewähren.
Tab. 4 Bewertung der Länge der Schichtblöcke und der Freiblöcke,

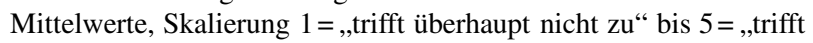
voll und ganz zu“"

\begin{tabular}{llll}
\hline Befragung & & $\begin{array}{l}\text { Mit der Länge } \\
\text { der Schichtblö- } \\
\text { cke in unserem } \\
\text { derzeitigen } \\
\text { Modell (d. h. } \\
\text { der Anzahl der } \\
\text { aufeinander } \\
\text { folgenden Ar- }\end{array}$ & $\begin{array}{l}\text { Mit der Län- } \\
\text { ge der Frei- } \\
\text { blöcke bin } \\
\text { ich zufrieden }\end{array}$ \\
beitstage) bin & \\
\hline ich zufrieden & \\
& & 4,20 & 4,12 \\
Zuftakt & Mittelwert & 49 & 49 \\
& $N$ & 0,676 & 0,781 \\
& Std.-Abweichung & $3,25^{\text {a }}$ & 4,26 \\
& Mittelwert & 53 & 53 \\
& $N$ & 0,998 & 0,858 \\
& Std.-Abweichung & 4,27 \\
& Mittelwert & $3^{\text {a }}$ & 49 \\
& $N$ & 48 & 0,785 \\
\hline
\end{tabular}

${ }^{\text {aSignifikanzniveau } 0,05}$

Die Auswertungen des Pilotjahres zeigen insgesamt, dass sinnvoller Weise nach einem Nachtdienst mindestens $48 \mathrm{~h}$, nach zwei und drei Nachtdiensten in Folge $72 \mathrm{~h}$ zur Erholung in Form von Freizeit zur Verfügung stehen sollten. Drei Nachtdienste in Folge kommen in den Grundplänen der Pilotdienststellen zwar nicht regelmäßig vor, doch in Vertretungsfällen oder durch die noch zu erbringenden $\mathrm{Zu}$ satzdienste (um auf die wöchentliche Arbeitszeit zu kommen) können drei Nachtdienste in Folge in der gelebten Praxis durchaus erbracht werden.

In der beispielgebenden Dienststelle sind die Befragten mit der Länge der Freiblöcke im pilotierten Modell zwischen drei und vier Tagen (je nach Lage der Zusatzschichten) sehr zufrieden, die Mittelwerte liegen deutlich über 4,0 auf der fünfteiligen Likert-Skala (vgl. Tab. 4).

Das Gegengewicht für längere Freiblöcke sind längere Schichtblöcke. Diese werden etwas schlechter bewertet als die Freiblöcke. Die Zustimmungswerte liegen für dieses Item bei üblicherweise sechs Schichttagen in Folge, also recht langen Schichtblöcken, bei einem Mittelwert von $\geq 3,0$, so dass dennoch von einem guten Wert gesprochen werden kann, auch wenn die Länge der Schichtblöcke im Vergleich zur Auftaktbefragung signifikant schlechter bewertet wird (vgl. Tab. 4).

Die Auswertungen der Schichtpläne zeigen, dass es gelingt, die Wochenendfreizeiten sicher zu stellen.

Diese Beobachtungen zum Verhältnis der Länge von Schichtblöcken zu Freiblöcken gelten auch für die anderen Pilotdienststellen. 
Abb. 2 Bewertung der Schlafqualität vor Frühdiensten

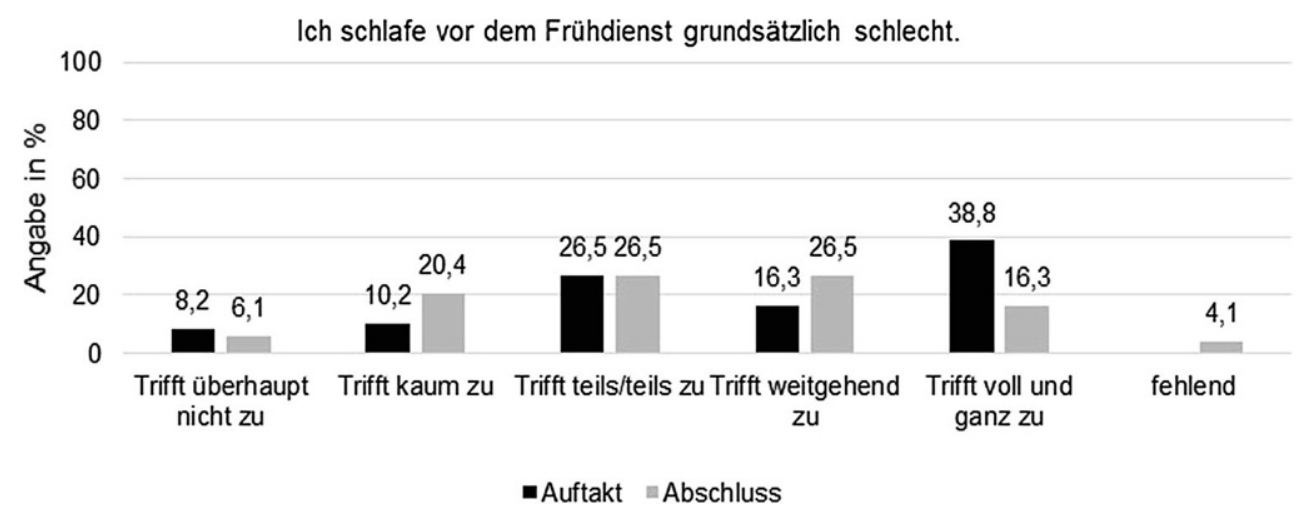

\subsubsection{Nicht mehr als drei Nachtschichten hintereinander}

Da die Ergebnisse aller Pilotdienststellen zur notwendigen Erholungszeit nach zwei bzw. drei Nachtschichten auf drei freie Tage hinweisen, ist es im Umkehrschluss unter Berücksichtigung der arbeitswissenschaftlichen Erkenntnisse erforderlich, bei der Planung maximal drei Nachtschichten in Folge nicht zu überschreiten.

Ein weiterer Hinweis für eine maximale Folge von drei Nachtschichten ergibt sich aus den Empfehlungen für eine schnelle Vorwärtsrotation: Wird diese strikt eingehalten, sind maximal drei Nachtschichten hintereinander im Grundplan sinnvoll.

\subsubsection{Schnelle Vorwärtsrotation}

Die Vorteile der Vorwärtsrotation drücken sich im Piloten vor allem in Schlafqualität und -quantität aus. Insbesondere die Frühschicht wird dann als sehr belastend wahrgenommen, wenn davor kein guter Schlaf möglich ist.

Die Ergebnisse der Befragungen zeigen, dass die Schlafqualität bzw. die Erholung in den Blockmodellen mit fünfbis sechstägiger Länge besser ist als in den kurz rückwärtsrotierenden Modellen oder im Doppelschlag.

In der hier betrachteten Dienststelle wird der Schlaf im Blockmodell eindeutig besser bewertet als im Doppelschlag (Abb. 2). Die Frage ist negativ formuliert, d.h. die Antwortkategorie ,trifft nicht zu“ bedeutet guter Schlaf vor Frühdiensten. In der Auftaktbefragung geben gut $55 \%$ der Antwortenden an, vor dem Frühdienst grundsätzlich schlecht zu schlafen (Summe aus „trifft weitgehend“ und „trifft voll

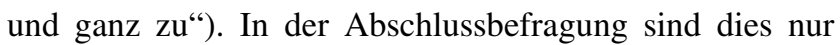
noch knapp $43 \%$ der Antwortenden!

\subsubsection{Länge der Schichten der Arbeitsbelastung anpassen}

Arbeitswissenschaftlich wird eine Schichtlänge von acht Stunden empfohlen. Grundsätzlich sollte die Schichtlänge an die Belastung, d.h. an die Arbeitsinhalte und Besonderheiten der Tätigkeit angepasst werden.
Vor der Pilotphase hat die ausgewählte Pilotdienststelle Spät- und Nachtschichten mit einer Länge von neun Stunden gearbeitet. Die Frühdienste waren sieben Stunden lang.

In Abb. 3 wird deutlich, dass die wahrgenommene Belastung aufgrund der Verkürzung der Nachtdienste von neun auf acht Stunden von Montag bis Sonntag signifikant sinkt.

Dieser positive Effekt der Nachtschichtverkürzung kann für alle Pilotdienststellen beobachtet werden, sowohl für Pilotdienststellen, die vor der Pilotphase im Doppelschlag gearbeitet haben, als auch für alle anderen. Es handelt sich also um einen offensichtlichen Effekt aus der Verkürzung der Nachtschichten.

Gleichzeitig steigt durch die Verlängerung der Frühdienste, bei der hier betrachteten Dienststelle von sieben auf acht Stunden, die wahrgenommene Belastung durch die Schichtlänge signifikant an (vgl. Abb. 4).

Der Spätdienst wird in der hier betrachteten Pilotdienststelle geringfügig belastender beschrieben als vor dem Piloten (vgl. Abb. 5). Dies kann möglicherweise darauf zurückgeführt werden, dass der Spätdienst nun nicht mehr die erste Schicht im Block ist und später beginnt. Durch den späteren Beginn startet die Spätschicht zu der Zeit, wo die Belastung durch die anfallenden Tätigkeiten bzw. Einsätze bereits höher ist (Spätdienst vorher neun Stunden, im Pilot acht Stunden; Beginn vor Pilot um 12 Uhr, im Pilot um 14 Uhr). Die Spätschicht ist im Polizeidienst an Wochentagen in der Regel die Schicht mit der höchsten Einsatzdichte.

Das bedeutet zusammengefasst, dass die wahrgenommenen Belastungen die verschiedenen Dienstzeiten betreffend offensichtlich sowohl durch die Schichtlänge als auch durch die Lage am Tag und im Block beeinflusst werden.

Grundsätzlich zeigen die Ergebnisse, dass eine durchschnittliche Länge von acht Stunden für die Schichtlängen in einem vorwärtsrotierenden Schichtmodell mit Schichtblöcken von fünf oder sechs Tagen sinnvoll sind und der Belastung entgegenwirken. Die niedrige Bewertung der Belastung durch die Länge der Nachtschichten wiegt die wahrgenommene höhere Belastung der Frühschichten auf. Die Entlastung der körperlich stark belastenden Nachtschichten ist aus arbeitswissenschaftlicher Sicht unbedingt zu fördern. 
Abb. 3 Belastung durch die Länge der Nachtdienste, Mittelwerte, Skalierung $1=$,trifft überhaupt nicht zu“ bis $5=$,trifft voll und ganz zu“, durchgängig acht Stunden, vorher neun Stunden Nachtdienst, Veränderungen signifikant, $p<0,05$
Abb. 4 Belastung durch die Länge der Frühdienste, Mittelwerte, Skalierung $1=$,trifft überhaupt nicht zu“" bis $5=$,trifft voll und ganz zu“", durchgängig acht Stunden im Pilot, vorher sieben Stunden Frühdienst, Veränderungen signifikant, $p<0,05$
Abb. 5 Belastung durch die Länge der Spätdienste, Mittelwerte, Skalierung $1=$ trifft überhaupt nicht zu bis $5=$ trifft voll und ganz zu, durchgängig acht Stunden im Pilot, vorher neun Stunden Spätdienst, nicht signifikant

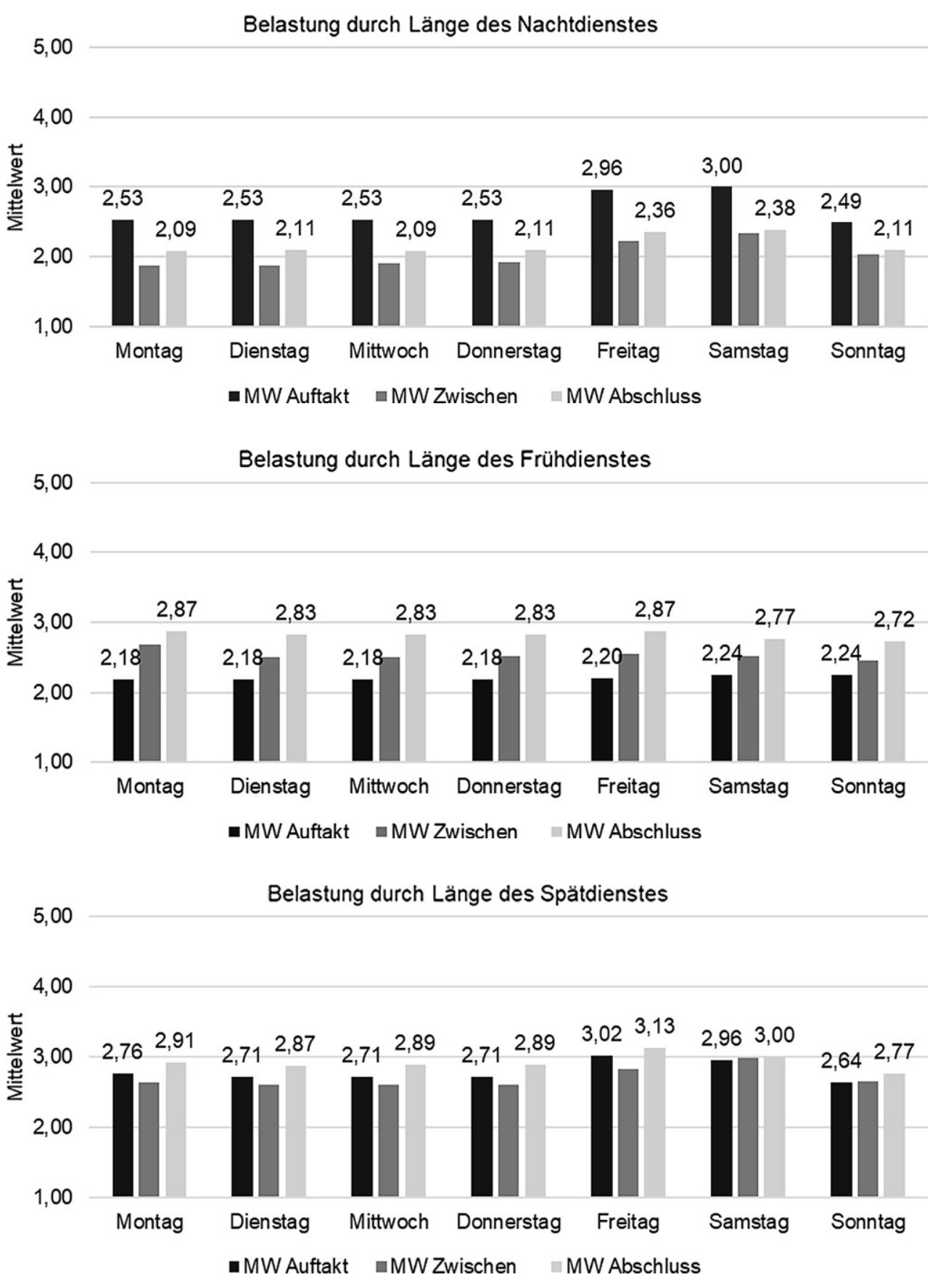

Insbesondere wenn dies parallel, wie beschrieben, mit einer Verbesserung der Schlafquantität und Schlafqualität vor der Frühschicht einhergeht.

Die Abb. 6 zeigt die Wahrnehmung der körperlichen Belastungen in allen drei Schichten im Vergleich. Die Spätschichten werden belastender wahrgenommen als die Nacht- und Frühschichten. Daher ist es dienststellenspezifisch sinnvoll, in Abhängigkeit einer Belastungsanalyse, die Spätschichten in der zweiten Hälfte durch vorgezogene Nachtschichten zu verstärken, wie es in der hier vorgestellten Pilotdienststelle praktiziert wird.

\subsection{Hohe Zufriedenheit mit den Modellen und gelingende Vereinbarkeit von Beruf und Privatleben}

Die Zufriedenheit mit den Blockmodellen zeigt, dass das Gleichgewicht zwischen Belastung und Entlastung, d.h. zwischen Arbeit und Freizeit erreicht wird. Die Vereinbarkeit von Beruf und Privatleben wird vor der Pilotphase mit einem Mittelwert von 3,65 bewertet und am Ende der Pilotphase mit einem Wert von 3,63. Offensichtlich gelingt die 
Abb. 6 Wahrgenommene körperliche Belastung in den verschiedenen Schichten, Skalierung $1=$ trifft überhaupt nicht $\mathrm{zu}$ bis $5=$ trifft voll und ganz zu, Belastung sinkt in Nachtschicht signifikant, Belastung in Frühschicht signifikant höher, $p<0,05$

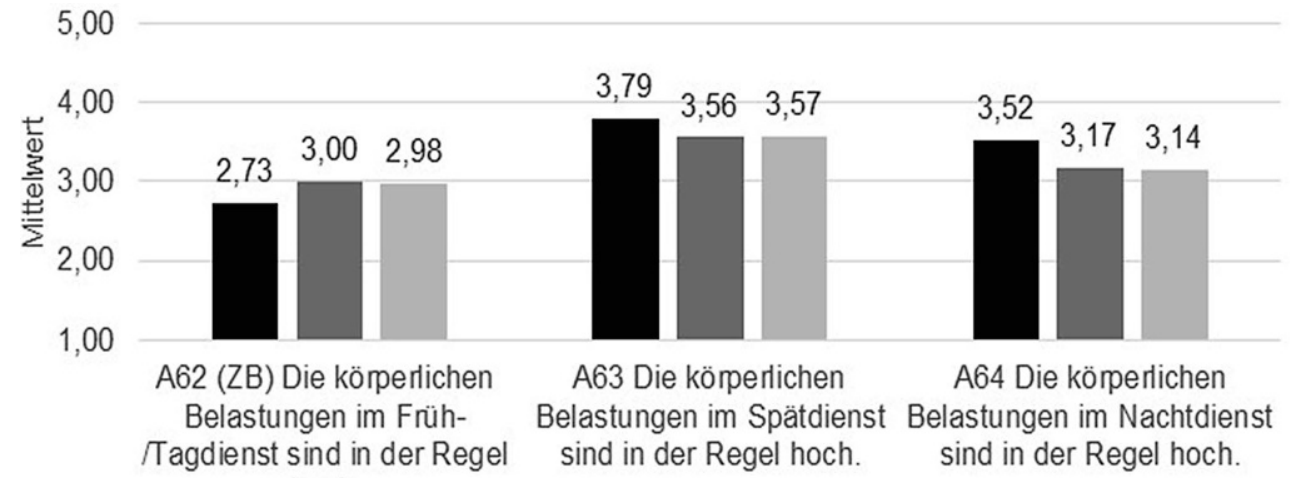

hoch.

-MW Auftakt घMWZwischen घMWAbschluss

Vereinbarkeit von Beruf und Privatleben im neuen Modell gleich gut.

Die modellimmanenten Freizeiten zwischen den Schichtarten (zwischen einer Früh- und Spätschicht sowie zwischen Spät- und Nachtschicht) betragen in den vorwärtsrotierenden Blockmodellen in der Regel 24h. Das führt zu einem unmittelbaren Ausgleich der Belastungen. Durch versetzte Schichten kann dieser Effekt verstärkt werden.

Dies zeigen die Ergebnisse für die hier betrachtete Dienststelle, in der die Schichtstärken entsprechend der Belastungsanalyse gebildet wurden.

Wie oben beschrieben, konnten durch die belastungsorientierten Besetzungen und versetzten Schichten in dieser Pilotdienststelle in besonderem Maße eine mitarbeiterorientierte Flexibilisierung und Individualisierung der Arbeitszeit im Schichtbetrieb realisiert werden. Die Zustimmung zur Teilnahme am Piloten bzw. zur Veränderung des Schichtmodells stieg in der Pilotphase stetig (Mittelwert vor Pilotphase 4,18; zum Ende der Pilotphase 4,58; nicht signifikant).

\subsubsection{Einschätzung der Gesundheit und Arbeitsfähigkeit}

Am Ende des Pilotjahres werden die körperlichen, zeitlichen und psychischen Arbeitsanforderungen sehr gut bewältigt; die Mittelwerte liegen alle deutlich über 3,5. Ergänzend steigt der $\mathrm{Spa} ß$ an der Arbeit, die Beamten und Beamtinnen fühlen sich deutlich ausgeruhter und leistungsfähiger mit dem neuen Schichtmodell (vgl. Abb. 7).

Ebenfalls deutliche Veränderungen sind, wie oben beschrieben, hinsichtlich Schlafqualität sowie der Erholung bzw. Entlastung für weitestgehend alle Dienststellen in Abhängigkeit der gewählten Modelle zu beobachten.

Der Work Ability Index (WAI) kam im Fragebogen zum Einsatz. Für 77 aller teilnehmenden Beamtinnen und Beamten konnte der WAI sowohl für die Auftakt- als auch die Abschlussbefragung berechnet werden. Die Werte zeigen keine signifikanten Veränderungen. Mit Werten zwischen
37 und 43 Punkten, maximal sind 49 Punkte möglich, verfügen diese Beamten und Beamtinnen über gute bis sehr gute Werte.

So kann insgesamt eine überaus erfolgreiche Pilotierung der arbeitswissenschaftlich empfehlenswerten und rechtskonformen Wechselschichtmodelle festgestellt werden.

\subsubsection{Weitere Einflussfaktoren über das Wechselschichtmodell hinaus}

Weitergehende Analysen der Gesamtdaten der Pilotphase im Rahmen einer sogenannten Faktorenanalyse zeigen die wesentlichen Einflussfaktoren auf „Gesünderes Arbeiten im Wechselschichtdienst". Neben der Zufriedenheit mit dem Schichtmodell als solchem, sind insbesondere die Belastungen aus den Früh-, Spät- und Nachtdiensten relevante Einflussgrößen. Darüber hinaus sind die wahrgenommene Personalausstattung, eindeutige Vertretungsregelungen und ein gesundes Führungsverhalten wichtige gestaltende Variablen, die auf die Gesundheit und Arbeitsfähigkeit im Wechselschichtdienst einzahlen. Mit anderen Worten sind dies ebenfalls entscheidende Stellschrauben für das gesündere Arbeiten im Wechselschichtdienst. Das bedeutet, dass neben den Schichtmodellen auch der ,weiche“ Rahmen der Schichtarbeit zu betrachten ist.

Daraus ergeben sich folgende Ansatzpunkte für eine gute Verhältnisprävention über die Gestaltung der Wechselschichtmodelle hinaus: Neben der sorgfältigen Prüfung des Verhältnisses von Mindest- zu Verfügungsstärken unter Berücksichtigung der Belastungen sollen auch dienststellenübergreifende Vertretungsregelungen entwickelt werden. Des Weiteren gilt es Dienststellenleiterinnen und -leiter sowie Dienstgruppenleiterinnen und -leiter mit erforderlichen Kompetenzen im Hinblick auf „gesunde“ Führung auszustatten. 
Abb. $7 \mathrm{Spaß}$ an der Arbeit, sich ausgeruht und leistungsfähig fühlen, Skalierung von $1=$,trifft überhaupt nicht $\mathrm{zu}^{\prime \prime}$ bis $5=$,trifft voll und ganz zu“, die Veränderungen sind nicht signifikant

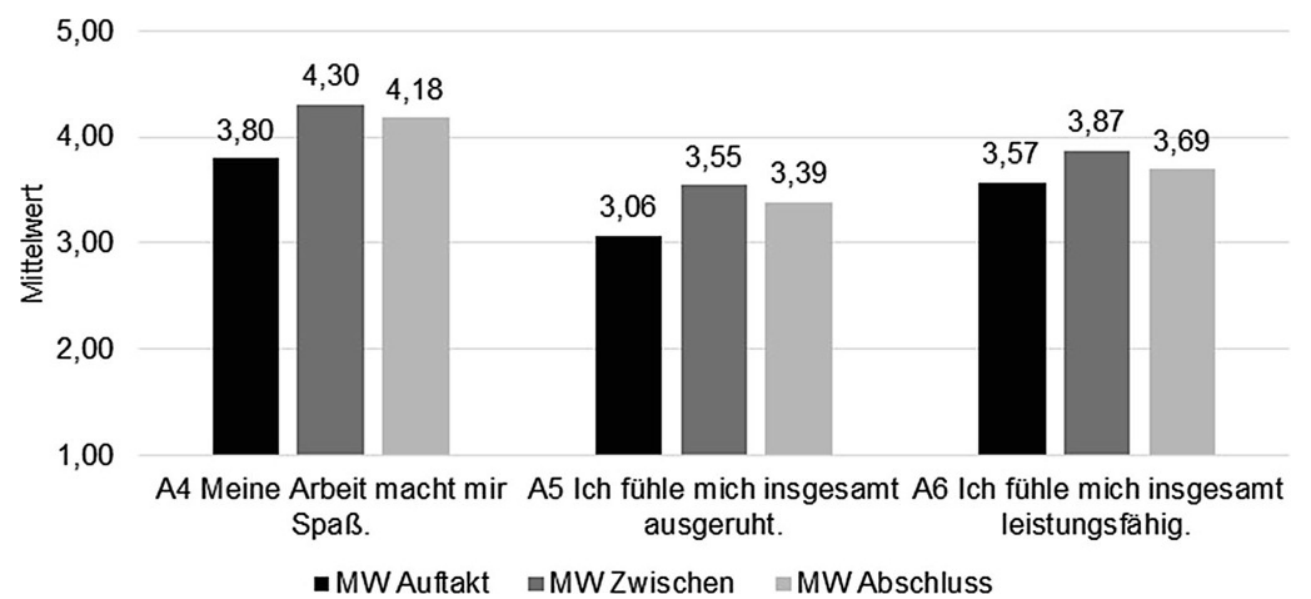

\subsection{Passung der neuen Modelle zu den dienstlichen Anforderungen}

Die Dokumentation der tatsächlich geleisteten Dienste aus den Hauptpilotdienststellen wurde entsprechend der oben beschriebenen Teilzielsetzungen ausgewertet. Die Ergebnisse zeigen folgendes:

- In der Pilotphase fanden so gut wie keine Schichttausche zwischen den Schichtgruppen statt, und es musste nur in wenigen Einzelfällen aus dem „Frei“ angeholt werden. So kann insgesamt von einer hohen Planungssicherheit gesprochen werden.

- Da alle Einsatzlagen bedient werden konnten und keine Unterstützung aus Nachbardienststellen angefordert werden musste, war die Leistungsfähigkeit und Effizienz des Wechselschichtdienstes in der Pilotphase gewährleistet.

- Sondereinsätze und Zusatzdienste konnten ebenfalls gestellt werden. Hierzu zählen beispielsweise Einsätze bei Fußballspielen, Demonstrationen und ähnlichen Großveranstaltungen.

- Vorrangig in den Nachtdiensten wurde auf Basis einer Belastungsanalyse mit versetzten Schichten experimentiert und an den Wochenenden wurde auf eine Orientierung an der Mindeststärke geachtet. So konnten die Einsätze zu den eher gesundheitsschädlichen Nachtzeiten und am Wochenende reduziert werden.

- Auch in den durch Zusatzdienste ergänzten Schichtplänen wurden die arbeitswissenschaftlichen Empfehlungen berücksichtigt und die Rechtskonformität sichergestellt.

Die Auswertungen zeigen, dass in den vorwärts rotierenden Modellen meist ausreichend Flexibilität vorhanden ist, die es ermöglicht mit dem vorhandenen Personal einen Teil des Personalbedarfs für Sondereinsätze, krankheitsbedingte Ausfälle etc. zu kompensieren, indem Schichten innerhalb einer Dienstgruppe verschoben werden können. Dies ist im Doppelschlag oder in reinen $12 \mathrm{~h}$ Modellen kaum möglich. Zusätzlicher Personalbedarf muss beim Doppelschlag oder
$12 \mathrm{~h}$ Modellen grundsätzlich aus einer der Dienstgruppen im Freiblock gedeckt werden, da ein zeitliches Verlängern oder Verschieben eines Dienstes oder ein Wechsel in eine andere Schicht kaum machbar ist.

Die Flexibilität in den Pilotdienststellen ist somit insgesamt gestiegen.

Die Auswertungen der Pläne zeigen weiter, dass es gelingt, Freizeit in möglichst langen Blöcken zu gewährleisten und nicht mehr als drei Nachtschichten hintereinander leisten zu müssen. Auch gelingt es, gesicherte Wochenendfreizeiten zu gewähren.

Fazit aus den qualitativen Rückmeldungen der verantwortlichen Planer und Planerinnen in den Pilotdienststellen ist, dass sich der Zugewinn an Flexibilität durch das Blockmodell positiv auf die Personalgestellung in Sondereinsätzen oder für Zusatzdienste bemerkbar macht.

\section{Zielerreichung}

\subsection{Zusammenfassung der Ergebnisse}

Das Monitoring der Dienstpläne zeigt für alle Pilotdienststellen, dass die Vorgaben der Arbeitszeitgesetzgebung (Rheinland-Pfalz und EU-Richtlinie) eingehalten und gleichzeitig Planungssicherheit und Leistungsfähigkeit des Wechselschichtdienstes weitestgehend sichergestellt werden können. Im Pilotjahr entstanden in den Pilotdienststellen keine Probleme bei der Besetzung von Sondereinsätzen und Zusatzdiensten.

Die Ergebnisse der Evaluation bestätigen insgesamt die Vorteilhaftigkeit von Wechselschichtmodellen, die arbeitswissenschaftliche Empfehlungen berücksichtigen und den rechtlichen Vorgaben uneingeschränkt, ohne Ausnahmen entsprechen.

Die Dienststelle, deren Ergebnisse aus der Pilotphase hier beschrieben werden, blieb nach der erfolgreichen Pilotierung bei diesem Modell. 
Die Auswertungen der Befragungen zeigen, dass die Freiblöcke zwischen den Schichtblöcken als ausreichend wahrgenommen werden. Der Mittelwert des Items zur Zufriedenheit mit der Länge der Freiblöcke ist zum Ende des Pilotjahres (Mittelwert 4,27) tendenziell höher als vor der Pilotphase (Mittelwert 4,12).

Durch die Vorwärtsrotation in Verbindung mit der Verkürzung der Schichtlängen der Nacht- und Spätschichten auf regelmäßig acht Stunden, wird eine signifikante Entlastung in den Nachtdiensten wahrgenommen.

Die körperlichen Belastungen in den Nachtschichten werden zum Ende der Pilotphase signifikant niedriger als vor der Pilotphase bewertet.

Die Spätschichten werden trotz der Verkürzung im Blockmodell geringfügig belastender empfunden als im Doppelschlag, was vermutlich an der Lage mitten im Block liegt. Im Doppelschlag war die Spätschicht die erste Schicht nach dem Freiblock, in die dann ausgeruht gestartet wurde.

Die wahrgenommene körperliche Belastung im Frühdienst steigt durch die Verlängerung auf acht Stunden signifikant an. Dies ist im Verhältnis zur erzielten Entlastung in den Nachtschichten nicht negativ zu sehen, denn insgesamt wird die Belastung in den Frühdiensten insgesamt niedriger als in den Nachtdiensten eingeschätzt. Weitere Entlastung wird offensichtlich auch durch die signifikant besser bewertete Schlafqualität vor den Frühschichten erzielt.

Insgesamt zeigt sich, dass es sinnvoll ist, die Länge der Schichten der Arbeitsbelastung anzupassen und durchschnittlich achtstündige Schichten in einem vorwärtsrotierenden Modell zu realisieren.

Das Blockmodell wird im Hinblick auf Belastung bzw. Beanspruchung sowie Vereinbarkeit von Beruf und Privatleben durchgängig gut bewertet. Gerade im Hinblick auf die Entlastung in den besonders belastenden Nachtschichten und die für die Gesundheit wichtige Schlafqualität, sind fünf- bis sechstägige Blockmodelle dem arbeitszeitmassierenden Doppelschlag unbedingt vorzuziehen.

\subsection{Umsetzung der Ergebnisse}

Die Bewertung der konkreten Gestaltungselemente der in der Pilotphase getesteten Schichtmodelle zeigt, dass es sinnvoll ist, bevor mit der Planung eines neuen Schichtmodells begonnen wird, eine Belastungsanalyse durchzuführen und die definierten Mindeststärken über den Tages- und Wochenverlauf zu prüfen. Dienststellenspezifisch sollte geprüft werden, wie die Belastung bzw. eigentliche Beanspruchung in der Spätschicht ,entschärft" werden kann. Entlastung kann beispielsweise durch versetzte Nachtschichten erreicht werden: Für einen Teil der Beamtinnen und Beamten beginnt die achtstündige Nachtschicht bspw. um 20 Uhr und endet bereits um vier Uhr, für einen an- deren Teil beginnt die Nachtschicht um 22 Uhr und endet um sechs Uhr. In der Zeit von vier bis sechs Uhr, in der körperlich sehr stark belastenden Zeit der Nachtschicht, ist die Dienststelle dann geringer besetzt. Auch versetzte Spätund Frühschichten können sinnvoll sein. Schichtplanvarianten mit versetzten Diensten setzen zwingend eine Prüfung der Mindeststärken voraus.

Ergänzend sollten die Bedarfe der Beamtinnen und Beamte der Dienststellen abgefragt werden und der Prozess partizipativ gestaltet werden.

Um die Einführung neuer Schichtmodelle gut zu unterstützen und langfristig ,gesünderes Arbeiten im Wechselschichtdienst“ zu ermöglichen, sollten Dienstgruppenleiterinnen und -leiter sowie Leiterinnen und Leiter der ,24/7Dienststellen“ in Sachen Führung mit Fokus auf dem Erhalt der Arbeitsfähigkeit qualifiziert werden. Das bedeutet, gemeinsam zu erarbeiten wie innerhalb des gesetzten Rahmens der Schutz der Gesundheit, das Sicherstellen von Qualifizierung, das (Vor-)Leben von gemeinsamen Werten und die Gestaltung einer entlastenden Arbeitsorganisation gelingen kann.

Das Land Rheinland-Pfalz hat die Ergebnisse der Pilotphase vollumfänglich in einer Verwaltungsvorschrift zur Gestaltung des Wechselschichtdienstes im November 2018 umgesetzt. Diese Vorschrift umfasst umfangreiche Regelungen, unter anderem ist in 2019 umzusetzen, dass

- für Nachtschichten grundsätzlich eine Länge von $8 \mathrm{~h}$ vorgesehen wird.

- ausschließlich vorwärtsrotierende Schichtmodelle mit maximalen Blocklängen von sechs Tagen zum Einsatz kommen.

- die Erholungszeiten unbedingt sichergestellt werden: Nach einem fünf- oder sechstägigen Schichtblock mit zwei Nachtschichten am Ende des Blockes sind unbedingt drei Tage frei zu halten.

Die Länge der Nachtdienste kann in einem Korridor von 8 bis 9 h Länge liegen, wenn dadurch der Spätdienst nachweislich entlastet wird. Und sonntags ist optional ein Nachtdienst mit $12 \mathrm{~h}$ Länge möglich. Eine vollständige Wiedergabe der Regelungen finden interessierte Leser und Leserinnen im Ministerialblatt der Landesregierung RheinlandPfalz (2018).

\section{Fazit}

Insgesamt liefert diese Studie wichtige Hinweise, wie die Überarbeitung oder Neugestaltung von Schichtmodellen in der Praxis, nicht nur im Polizeidienst, gelingen kann. Sie zeigt, dass auch oder gerade für den Polizeidienst, der häufig mit nicht planbaren Ereignissen während der Arbeitszeit einhergeht, arbeitswissenschaftlich gute und rechtskonfor- 
me Modelle mit Entlastung einhergehen und die Planungssicherheit verbessern.

Die Nachteile der Studie bestehen vor allem in Größe und Struktur der Stichprobe. Die Gesamtstichprobe Beamten und Beamtinnen ist ausreichend groß und erfüllte die Anforderungen an die gängigen statistischen Verfahren. Da sich diese Stichprobe auf insgesamt sieben Dienststellen verteilt und jede dieser Dienststellen einen anderen Schichtplan testete (was politisch gewollt war), waren dann die einzelnen Stichprobengrößen in den Dienststellen recht klein. Dies hat zur Folge, dass Auswertungen über soziodemografische Daten auf Dienststellenebene nicht mehr möglich waren, um die Anonymität nicht in Frage zu stellen.

Ergänzend könnten physiologische Längsschnittuntersuchen weitere Erkenntnisse über die Belastungen bzw. Entlastungen in unterschiedlichen Schichtmodellen aufzeigen.

Open Access Dieser Artikel wird unter der Creative Commons Namensnennung 4.0 International Lizenz (http://creativecommons.org/ licenses/by/4.0/deed.de) veröffentlicht, welche die Nutzung, Vervielfältigung, Bearbeitung, Verbreitung und Wiedergabe in jeglichem Medium und Format erlaubt, sofern Sie den/die ursprünglichen Autor(en) und die Quelle ordnungsgemäß nennen, einen Link zur Creative Commons Lizenz beifügen und angeben, ob Änderungen vorgenommen wurden.

\section{Literatur}

Bundesanstalt für Arbeitsschutz und Arbeitsmedizin Gestaltungsempfehlungen für Nacht- und Schichtarbeit. https://www.baua. de/DE/Themen/Arbeitsgestaltung-im-Betrieb/Arbeitszeit/Nachtund-Schichtarbeit.html. Zugegriffen: 27. Febr. 2018
Bürger B (2015) Arbeitszeitmodelle für den Streifendienst der Polizei. Eine interdisziplinäre Analyse am Beispiel der Bayerischen Polizei. Verlag für Polizeiwissenschaft, Frankfurt am Main

DGUV (2012) Report 1/2012: Schichtarbeit - Rechtslage, gesundheitliche Risiken und Präventionsmöglichkeiten. Deutsche Gesetzliche Unfallversicherung, Berlin, Rheinbreitbach

Europäische Union (2003) RICHTLINIE 2003/88/EG DES EUROPÄISCHEN PARLAMENTS UND DES RATES vom 4. November 2003 über bestimmte Aspekte der Arbeitszeitgestaltung. https://eur-lex.europa.eu/legal-content/DE/TXT/PDF/? uri=CELEX:32003L0088\&from=EN (Erstellt: 18. Nov. 2003). Zugegriffen: 25. Apr. 2019

Knauth P, Hornberger S (1987) Schichtarbeit und Nachtarbeit Bd. 4. Bayerisches Staatsministerium für Arbeit und Sozialordnung, Familie, Frauen und Gesundheit, Wolznach (Probleme - Formen Empfehlungen)

Ministerialblatt der Landesregierung Rheinland-Pfalz 2018, 70. Jg, Nr. 9

Nachreiner F (1978) Über Determinanten der Einstellung zur Schichtarbeit. Z Arb Wiss 34(I):6-11

Rothe I, Beermann B, Wöhrmann AM (2017) Arbeitswissenschaftliche Erkenntnisse zur Arbeitszeit und Gesundheit. In: Schröder L, Urban H-J (Hrsg) Gute Arbeit 2017. Streit um Zeit - Arbeitszeit und Gesundheit. Bund-Verlag, Frankfurt am Main

Rutenfranz J, Knauth P, Nachreiner F (1993) Arbeitszeitgestaltung. In: Schmidtke H (Hrsg) Ergonomie. Carl Hanser, München, Wien

Süs J, Weitershagen C, Rausch M, Watrinet C, Mündlein S (2018) Abschlussbericht Pilotprojekt Gesünderes Arbeiten im Wechselschichtdienst. Leitung der Arbeitsgruppe Gesünderes Arbeiten im Wechselschichtdienst der Polizei Rheinlandpfalz (Hrsg). Koblenz, Internes Arbeitspapier

Spencer M, Robertson K, Folkard S (2006) The development of a fatigue/risk index for shiftworkers. http://www.hse.gov.uk/research/ rrpdf/rr446.pdf. Zugegriffen: 25. Apr. 2019 